\title{
Comparison of multi-omics results between patients with acute myeloid leukemia with long-term survival and healthy controls
}

\author{
Yang Song, Qishan Hao, Guangji Zhang, Qiuyun Fang, Zhe Wang, Yan Li, Hui Wei, Ying Wang, \\ Erlie Jiang, Zheng Tian, Yannan Jia, Min Wang, Jianxiang Wang, Yingchang Mi \\ State Key Laboratory of Experimental Hematology, National Clinical Research Center for Blood Diseases, Institute of Hematology and Blood \\ Diseases Hospital, Chinese Academy of Medical Sciences \& Peking Union Medical College, Tianjin, China \\ Contributions: (I) Conception and design: Y Song, Y Mi; (II) Administrative support: J Wang, M Wang, Y Mi; (III) Provision of study materials or \\ patients: G Zhang, Q Fang, Y Li, E Jiang, H Wei, Y Wang; (IV) Collection and assembly of data: Z Tian; (V) Data analysis and interpretation: Q \\ Hao, Z Wang, Y Jia; (VI) Manuscript writing: All authors; (VII) Final approval of manuscript: All authors. \\ Correspondence to: Professor Yingchang Mi. State Key Laboratory of Experimental Hematology, National Clinical Research Center for Blood \\ Diseases, Institute of Hematology and Blood Diseases Hospital, Chinese Academy of Medical Sciences \& Peking Union Medical College, Nanjing \\ Rd. 288, Tianjin 300020, China. Email: ychmi@ihcams.ac.cn.
}

Background: Acute myeloid leukemia (AML) is a group of highly heterogeneous diseases, for which approximately $35-40 \%$ of patients younger than 60 years old can be cured. However, the multi-omics characteristics and immune cell infiltration (ICI) status of adult long-term survival patients with AML patients compared with healthy controls are still relatively under-explored.

Methods: A total of 10 healthy transplant donors (control group) and 11 long-term survival patients with AML with de novo sampling from 2019 to 2020 at the Institute of Hematology in the Hospital of Blood Diseases were enrolled. We simultaneously performed $850 \mathrm{~K}$ methylation and bulk RNA-seq on these 21 patients for comparing the differential gene methylation and expression levels between the two groups. The analysis of immune cell gene expression was based on 4 algorithms single sample gene set enrichment analysis (ssGSEA), EPIC, ESTIMATE and immunophenotype score (IPS) on the bulk RNA-seq data.

Results: The differential methylation positions (DMPs) of the control group was significantly higher than that of the long-term survival group $(\mathrm{P}<0.01)$. The hypomethylated probes for Kyoto Encyclopedia of Genes and Genomes (KEGG) enrichment is summarized as follows: the significant pathway was related to NK-cellmediated cytotoxicity and amino acid metabolism. We also found the Differential expression genes (DEGs) of long-term survival AML were roughly similar, and the DEGs were highly relevant to the cellular amino acid metabolic process pathway by gene set enrichment analysis (GSEA). Based on the further univariate and multivariate Cox survival analyses in GSE37642, genes crosslinked of DEGs and DMPs: LOXL1 and PDZRN4, which characterized as hypomethylated and upregulated, may become an AML prognostic marker $(\mathrm{P}<0.05)$. Besides, compared with the long-term-survival AML patients who discontinued chemotherapy after $>3$ years and the healthy donors, T cell-, natural killer cell-, MHC- and effector cell (EC)-related genes were downregulated; suppressor cells (SC) and checkpoint (CP) cells were significantly upregulated in the long-term-survival AML patients who discontinued chemotherapy after $<3$ years.

Conclusions: In terms of DNA methylation, RNA expression and ICI, AML patients with long-term survival were slightly different than that of healthy people. The profile of long-term AML survivors, especially those who discontinued chemotherapy less than 3 years, still differed from that of healthy people.

Keywords: Acute myeloid leukemia (AML); immune cell infiltration (ICI); long-term survival; RNA-seq

Submitted Nov 23, 2021. Accepted for publication Jan 13, 2022.

doi: 10.21037/atm-21-6681

View this article at: https://dx.doi.org/10.21037/atm-21-6681 


\section{Introduction}

Acute myeloid leukemia (AML) is a group of highly heterogeneous diseases, for which approximately $35-40 \%$ of patients younger than 60 years old can be cured (1). Clinically, patients with AML in complete remission (CR) for $>3$ years and measurable/minimal residual disease (MRD)-negative can be considered as 'long-term survival patients' (1).

The concept of immune cell infiltration (ICI) was derived from quantitative assessment of increased tumorinfiltrating lymphocytes (TILs), which is highly related to the tumorigenesis, development, metastasis and mortality of solid tumors (2).

Great effort has been made to explore the significant markers associated with an unfavorable prognosis of AML patients $(3,4)$. Recently, Wang et al. provided an immune risk score model for predicting the survival of patients with AML. The immune cells enumerated by CIBERSORT algorithm: monocytes, resting mast cells, eosinophils, CD8 + positive T cells, resting NK cells, resting dendritic cells and resting memory CD $4+\mathrm{T}$ cells were the most representative cells in the bone marrow microenvironment of AML (3). Dong et al. integrated the multi-omics in terms of drug sensitivity, signaling pathways and ICI in AML from the viewpoint of public transcriptional profile mining (4).

However, no one has evaluated the immune cell proportions in different AML status. To improve knowledge of the characteristics of adult long-term survival patients with AML, we mainly focused on the multi-omics (DNA methylation and gene expression) difference of long-term survival AML population and healthy donors. In addition, the ICI status of AML patients compared with healthy controls were explored.

We present the following article in accordance with the MDAR reporting checklist (available at https://atm. amegroups.com/article/view/10.21037/atm-21-6681/rc).

\section{Methods}

\section{Patients}

A total of 10 healthy transplant donors (control group) and 11 long-term survival patients with AML with de novo sampling from 2019 to 2020 at the Institute of Hematology in the Hospital of Blood Diseases were enrolled. The longterm survival patients were divided into two groups: those who discontinued chemotherapy after $>3$ or $<3$ years.
Written informed consent was given by all patients, who were in sustained remission for $>3$ years until the last follow-up on August 30, 2021. The study was conducted in accordance with the Declaration of Helsinki (as revised in 2013). The study was approved by institutional committee of Hematology and Blood Diseases Hospital, Chinese Academy of Medical Sciences (No. KT2018091-EC-2).

\section{DNA methylation and $R N A-s e q$ analysis}

We simultaneously performed $850 \mathrm{~K}$ methylation and bulk RNA-seq for these 21 study subjects. R packages 'ChAMP' and 'DEseq2' were used for differential analysis. We defined genes with $\mid \log \mathrm{FCl} \geq 1$ and adjusted $\mathrm{P}<0.05$ as differential expression genes (DEG). Moreover, genes with I delta $\beta \mid \geq 0.1$ and adjusted $\mathrm{P}<0.05$ were designated differential methylation positions (DMP).

\section{ICI analysis}

Based on the bulk RNA-seq data, a reference was made based on the definition of 27 immune cell types in solid tumor (2). The single sample gene set enrichment analysis (ssGSEA) (4), CIBERSORT (5) and EPIC (6) algorithms were used to quantify the immune cell-related gene expression in the bone marrow microenvironment, and cell types irrelevant to AML immunity (i.e., "Blood vessels", "Normal mucosa", "SW480 cancer cells" and "Lymph vessels") were excluded. ESTIMATE (7) was used to evaluate the immune and stromal contents of AML bone marrow environment.

The immunophenotype score (IPS) algorithm (8) is a panel of immune cell-related genes used to predict the immunotherapy response and consists of four clusters: major histocompatibility complex (MHC)-related molecules, effector cells (EC), suppressor cells (SC) and checkpoint cells (CP). The z-score for each sample was calculated by the weighted averaged z-score of four cluster values requiring the corresponding gene expressions and their weights.

\section{Gene Expression Omnibus (GEO) dataset mining}

GEO database RNA-seq expression data (accession No. GSE 37642) through PubMed was used for selected gene survival analysis. Totally, 531 AML patients with clinical information and RNA expression were extracted. The expression profile fragments per kilobase per million was 
Table 1 Clinical characteristics of study cohort

\begin{tabular}{|c|c|c|c|c|c|c|c|}
\hline No. & Sex & Age (years) & FAB & Outcome & $\begin{array}{c}\text { Stop chemo till } \\
\text { sampling time (months) }\end{array}$ & $\begin{array}{l}\text { DFS till sampling } \\
\text { time (months) }\end{array}$ & $\begin{array}{l}\text { OS (months) } \\
\text { until now }\end{array}$ \\
\hline 1 & $M$ & 39 & M2 & Alive & 36 & 42 & 68 \\
\hline 2 & $\mathrm{~F}$ & 61 & M2 & Alive & 36 & 42 & 67 \\
\hline 3 & M & 52 & M5 & Alive & 36 & 42 & 76 \\
\hline 6 & M & 58 & M5 & Alive & 50 & 58 & 83 \\
\hline 7 & $F$ & 40 & M2 & Alive & 37 & 43 & 71 \\
\hline 8 & M & 67 & M5 & Alive & 31 & 38 & 63 \\
\hline
\end{tabular}

F, female; M, male; FAB, French-American-British classification; OS, overall survival; DFS, disease-free survival.

converted into transcripts per kilobase million and then "Combat" was used to remove the batch effect.

\section{Statistical analysis}

The statistical data analysis in this study was performed using $\mathrm{R}$ version 3.6.2. Significance was determined using the Mann-Whitney test, and all $\mathrm{P}$ values were two-sided and considered statistically significant when $<0.05$.

\section{Results}

\section{Clinical data}

The 11 patients with AML with long-term survival comprised 6 men and 5 women, with a median age of 43 [2667] years. Among them, 7 patients completed chemotherapy for at least 3 years (discontinued chemotherapy after $>3$ years) and 4 completed chemotherapy in $<3$ years (discontinued chemotherapy after $<3$ years). All patients achieved CR after one course of induction chemotherapy, followed by 3-6 courses of consolidation cycle treatment. None of them received allogeneic hematopoietic stem cell transplantation. The median overall survival (OS) and disease-free survival (DFS) till sampling time were 42 [19-58] and 36 [15-50] months, respectively (Table 1). A total of 10 healthy people were transplant donors ( 7 men, 3 women), with a median age of 43 [22-56] years.

\section{Multi-omics differences}

We detected 1,152 DMPs: 1,092 hypomethylated probes and 60 hypermethylated probes (Figure 1A). The methylation level of the control group was significantly higher than that of the long-term survival group $(\mathrm{P}<0.01)$ (Figure 1B). The hypomethylated probes for Kyoto Encyclopedia of Genes and Genomes (KEGG) enrichment are summarized as follows: the significant pathway was related to NK-cell-mediated cytotoxicity and amino acid metabolism (Figure 1C), whereas no signaling pathway was found to be enriched by hypermethylated probes.

Next, we analyzed the DEGs between these two groups: 651 genes upregulated and 142 genes downregulated in the long-term survival AML group (Figure $2 A$ ). We found the DEGs of long-term survival AML were roughly similar, and the DEGs were highly relevant to the cellular amino acid metabolic process pathway by GSEA (Figure $2 B$ ).

We crosslinked the methylation data and RNA profiles of the controls and patients. Comparing with healthy people, 8 genes (LOXL1, CFI, RHBDF1, RFPL2, NCR3, DNM1, ARHGAP6 and PDZRN4) were hypomethylated and upregulated, gene CRISP2 was hypermethylated and downregulated, 3 genes (PDGFRA, CHST1, and MMRN1) were hypomethylated and downregulated, CCR10 was hypermethylated and upregulated in the long-term survival AML patients with a threshold of I delta beta $\mid>0.1$, and $\mid \log \mathrm{FCl}>1$ (Figure 2C). 
A

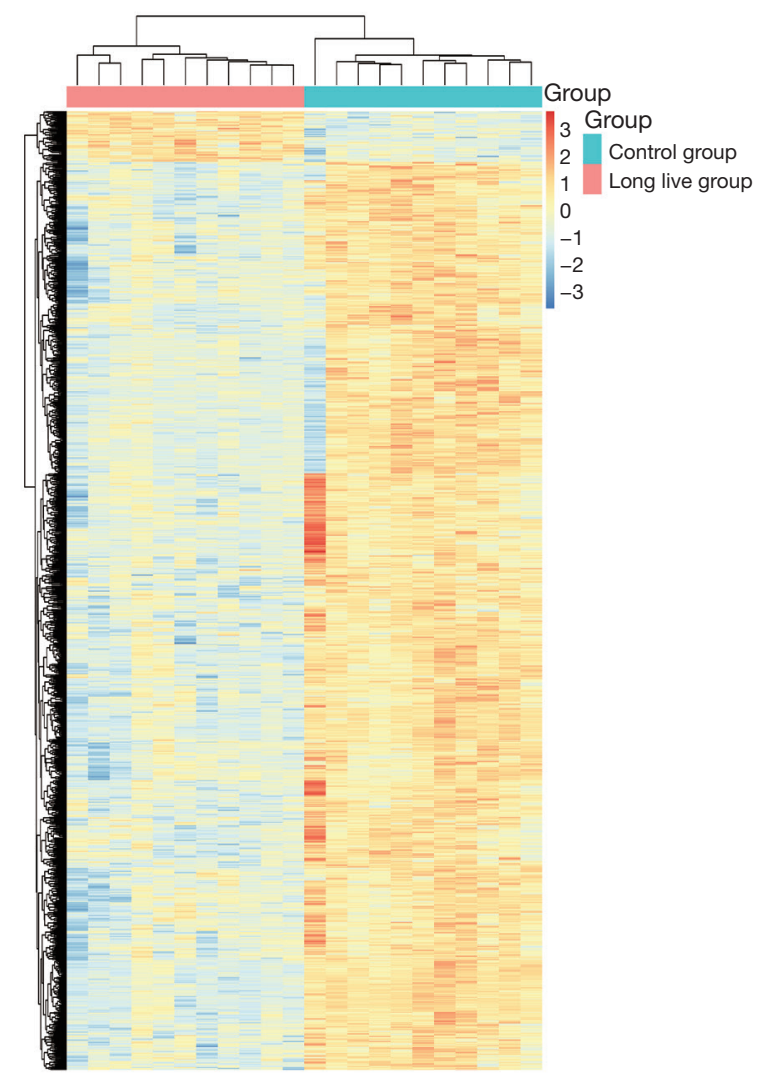

B

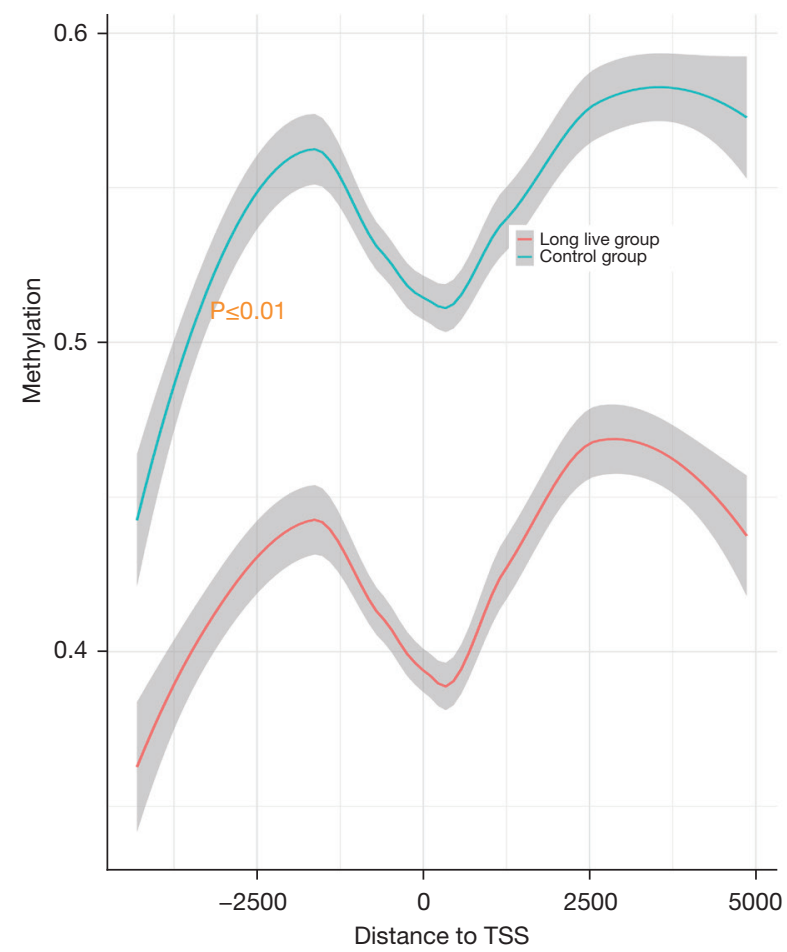

c

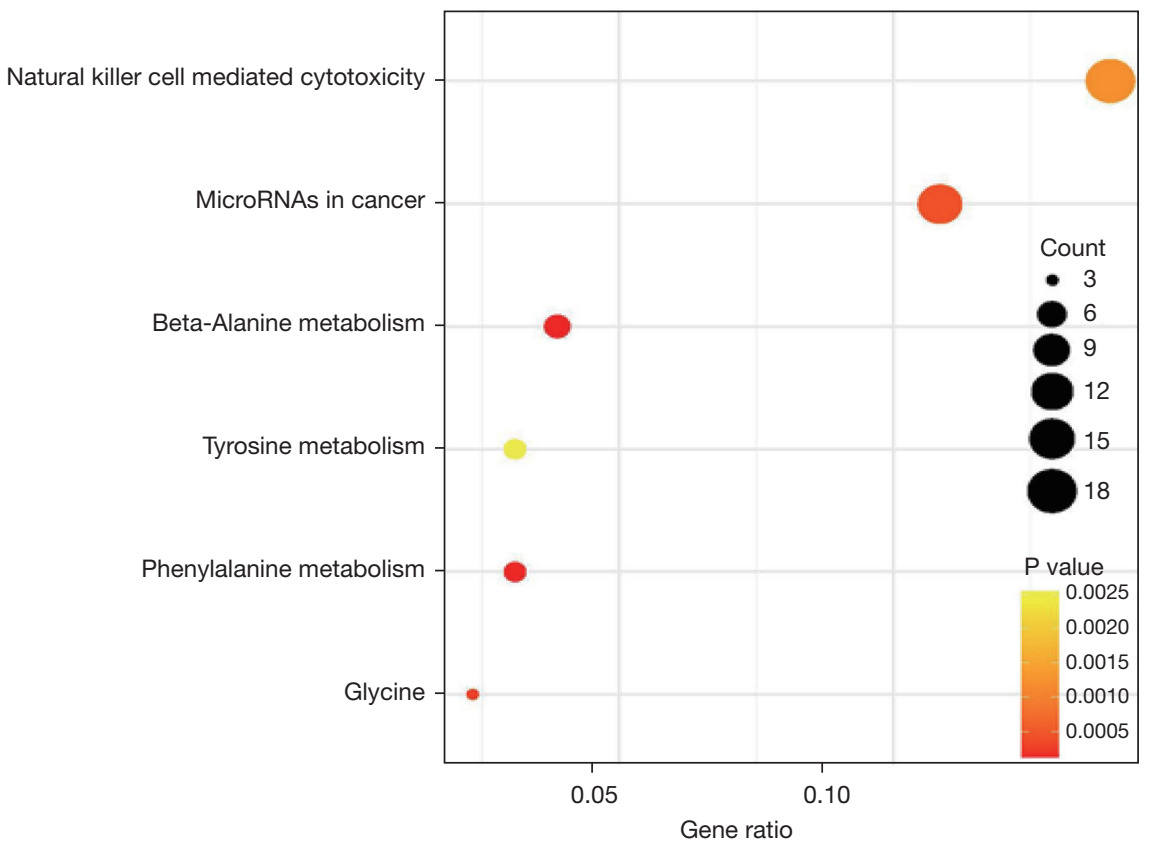

Figure 1 The comparison of long-term survival acute myeloid leukemia (AML) group and healthy Control group in DNA level. (A) The differential methylation position (DMP) heatmap of long-term survival (Long Live) Group and Control Group comparison; (B) extracting DMPs from each CpG island for the long-term survival (Long Live) group and methylation comparison. $\mathrm{X}$ : distance to the transcription start site (TSS); Y: methylation level ranging [0-1]. (C) KEGG pathway enrichment for the long-term survival group's upregulated genes. 


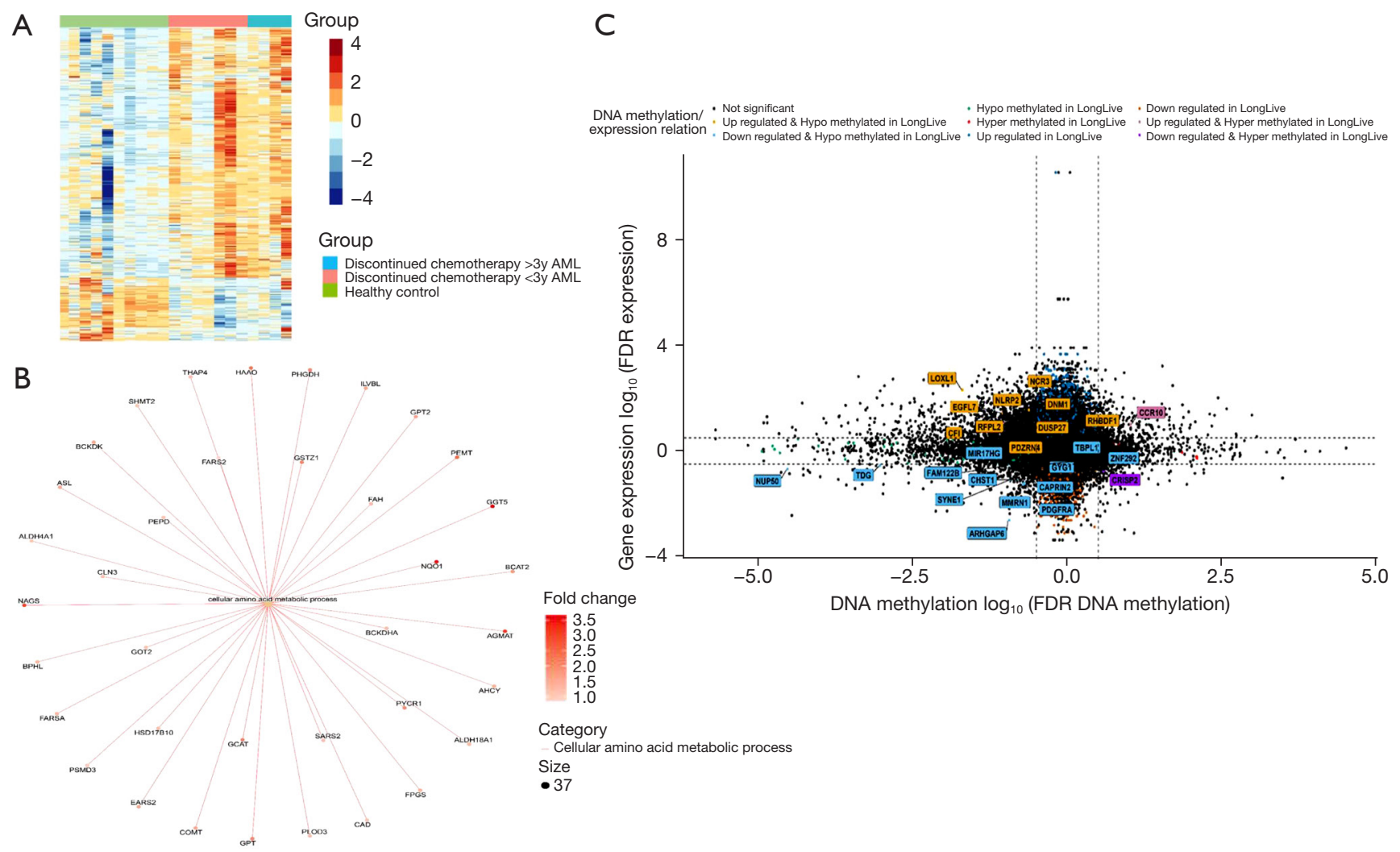

Figure 2 The crosslink analysis of long-term survival acute myeloid leukemia (AML) group and healthy Control group. (A) RNA-seq gene expression heatmap of the long-term survival AML group and healthy Control group comparison. (B) Enrichment Gene Ontology (GO) plot for the cellular amino acid metabolic process and its related genes. (C) Starburst plot showing the crosslink analysis of DNA methylation and RNA expression relation (healthy people $v s$. long-term survival AML patients).

To identify the significance of DEGs and DMPs between the groups, we put these crosslink genes into the GEO database for further exploration of the prognostic significance of the genes.

The univariate Cox analysis results of the signature for GEO: hazard ratio (HR) of LOXL1 was 1.12 with $95 \%$ confidence interval (CI) from 1.024 to $1.217(\mathrm{P}=0.011848)$; HR of PDZRN4 was 1.34 with $95 \%$ CI from 1.05 to 1.7 $(\mathrm{P}=0.017353)$; HR of MMRN1 was 1.08 with $95 \% \mathrm{CI}$ from 1.00 to $1.16(\mathrm{P}=0.036575)$; HR of CFI was 1.20 with $95 \%$ CI from 1.01 to $1.44(\mathrm{P}=0.042251)$.

The multivariate Cox analysis results of the signature for GEO: HR of LOXL1 was 1.140 with $95 \%$ CI from 1.045 to $1.245(\mathrm{P}=0.003)$; HR of PDZRN4 was 1.246 with $95 \%$ CI from 1.009 to $1.582(\mathrm{P}=0.028)$.

Based on the univariate and multivariate Cox analyses, two crosslinked genes LOXL1 and PDZRN4 (Table 2) characterized as hypomethylated and upregulated, may become an AML prognostic marker $(\mathrm{P}<0.05)$.

\section{ICI landscape}

To investigate the ICI difference between the healthy controls and long-term survival patients with AML, we separately calculated the immune cell gene expression by ssGSEA, EPIC, ESTIMATE and IPS algorithms (Figure $3 A$ ).

The results of ssGSEA analysis suggested that the immune cell proportion and related gene expression status of patients discontinuing chemotherapy for $>3$ years were extremely similar to those of healthy people, except for the NK-CD56 bright cells ( $\mathrm{P}=0.016)$, which were highly downregulated in patients with AML who completed chemotherapy for $>3$ years. In contrast, compared with the healthy controls, there was more infiltration of NK cells, gamma delta $\mathrm{T}$ cell and CD8 $\mathrm{T}$ cells, and less of 
Table 2 Univariate and multivariate Cox analyses of crosslinked genes in GEO cohort

\begin{tabular}{|c|c|c|c|c|}
\hline Gene & \multicolumn{2}{|c|}{ Univariate analysis } & \multicolumn{2}{|c|}{ Multivariate analysis } \\
\hline LOXL1 & $1.12(1.024-1.217)$ & 0.011848 & $1.14(1.045-1.245)$ & 0.003 \\
\hline PDZRN4 & $1.34(1.05-1.70)$ & 0.017353 & $1.246(1.009-1.582)$ & 0.028 \\
\hline MMRN1 & 1.08 (1.00-1.16) & 0.036575 & $1.085(0.982-1.168)$ & 0.107 \\
\hline DNM1 & $0.92(0.85-1.01)$ & 0.080694 & - & - \\
\hline NCR3 & $0.77(0.55-1.08)$ & 0.127044 & - & - \\
\hline PDGFRA & $1.17(0.78-1.77)$ & 0.438077 & - & - \\
\hline CRISP2 & $1.06(0.90-1.25)$ & 0.485599 & - & - \\
\hline CCR10 & $1.05(0.80-1.38)$ & 0.710786 & - & - \\
\hline RHBDF1 & $0.97(0.80-1.17)$ & 0.755652 & - & - \\
\hline RFPL2 & $0.98(0.76-1.25)$ & 0.848194 & - & - \\
\hline
\end{tabular}

GEO, Gene Expression Omnibus; HR, hazard ratio; Cl, confidence interval.

eosinophils, neutrophils, Tem (effector memory $\mathrm{T}$ cells), $B$ cells and macrophages in patients who discontinued chemotherapy after $<3$ years.

Then, the IPS score quantitatively evaluated the immunotherapeutic response of these two patient groups applying the four main broadly similar clusters of $\mathrm{MHC}$, $\mathrm{SC}, \mathrm{CP}$ and EC (Figure 3B). The immune cell-related gene expression profile of patients with $\mathrm{AML}$ who discontinued chemotherapy after $>3$ years was roughly similar to that of the normal controls. Statistical differences were only observed in immune CPs PD-1 $(\mathrm{P}=0.025)$ and LAG3 $(\mathrm{P}=0.025)$ and ECs CD8 $(\mathrm{P}=0.01)$ and temCD4 $(\mathrm{P}=0.025)$. Generally, the long-term survival AML patients and the controls showed different immune cell patterns (Figure 3C).

Furthermore, we separately analyzed the relationship between crosslinked prognostic genes and the immune cell proportion. Our results suggested the two groups presented different infiltration patterns of crosslinked genes (Figure $4 A, 4 B$ ). It worth noting that PDZRN4 tended to have a negative correlation with immune cells, especially the $\mathrm{B}$ cell and $\mathrm{T}$ cell family $(\mathrm{P}<0.01)$, in the long-termsurvival AML patients who discontinued chemotherapy after $<3$ years, in contrast to no significant relationship in long-term-survival AML patients who discontinued chemotherapy after $>3$ years and the healthy controls.

\section{Discussion}

Currently, an explanation for the difference between long-term survival patients with AML and healthy people remains unclear. One way to examine this problem is to compare the similarities and differences of bone marrow hematopoietic cells and immune microenvironments between these two groups of patients.

The immune cell subpopulation estimation was horizontally contrasted in our long-term survival AML cohort based on different algorithms (2,5-9). The wholeimmune phenotype results roughly agreed with these algorithms.

The functional roles of bone marrow cells show various capabilities: cytotoxic lymphocytes and NK cells have antitumor effects; macrophages and neutrophils possess both tumor-promoting and antitumor effects; myeloid derived SCs, regulatory $\mathrm{T}$ cells and mast cells are recognized as tumor-promoting. Interestingly, there was less infiltration of both $\mathrm{T}$ cells and NK cells in patients with AML who discontinued chemotherapy after $<3$ years. DMP hypomethylation probe enrichment was also consistently associated with the NK-cell cytotoxicity pathway.

The IPS score was utilized to evaluate the immunotherapeutic response. MHC, EC, SC and CP 
A

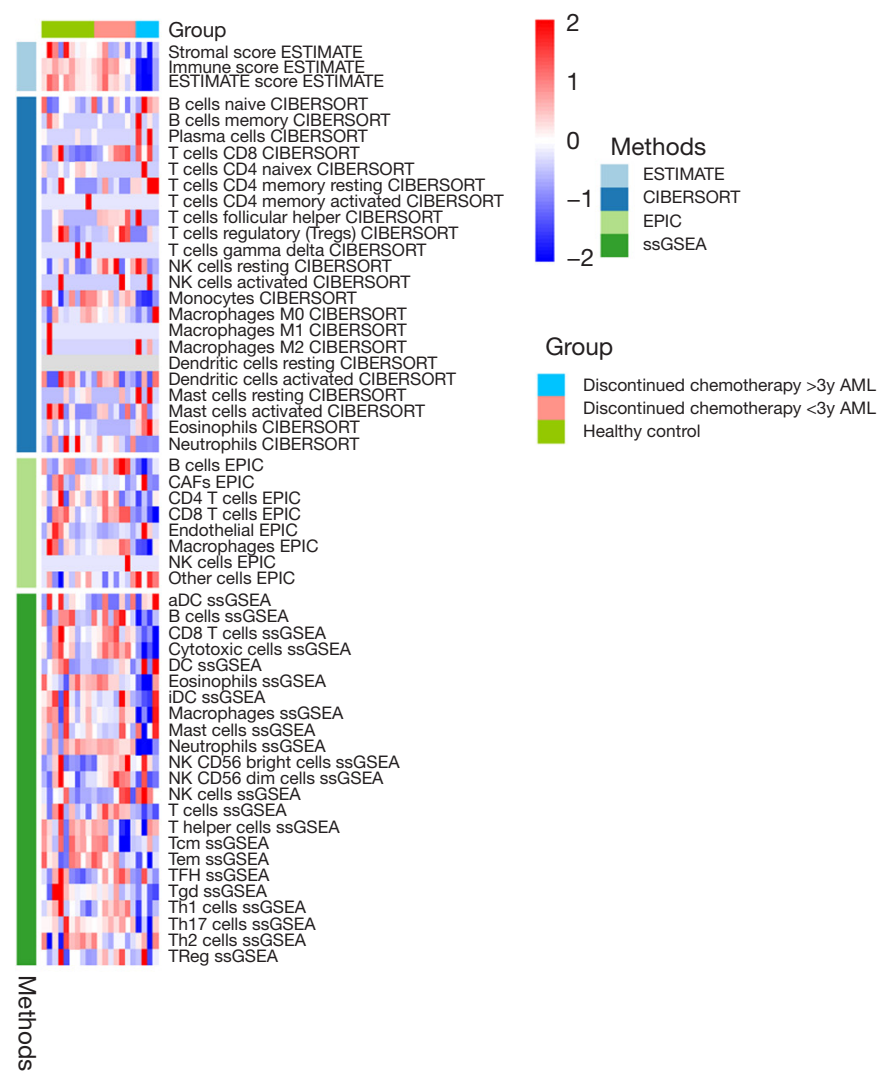

B

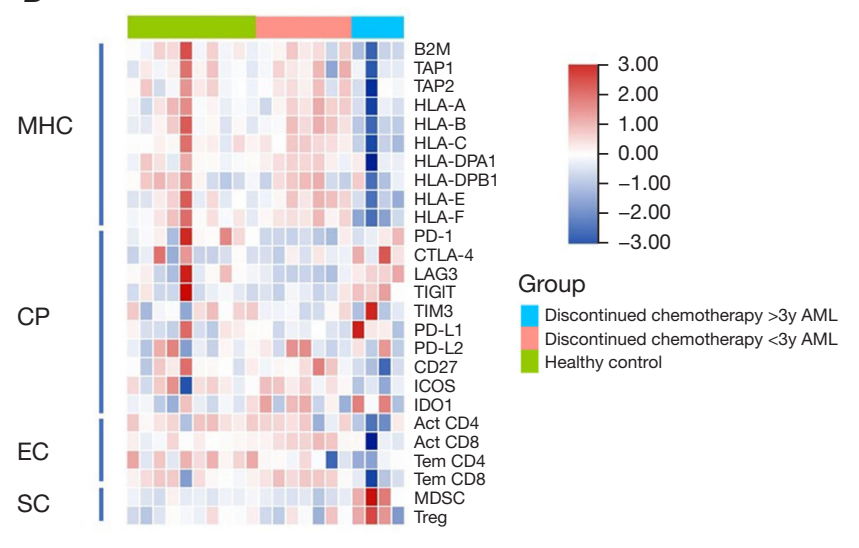

C

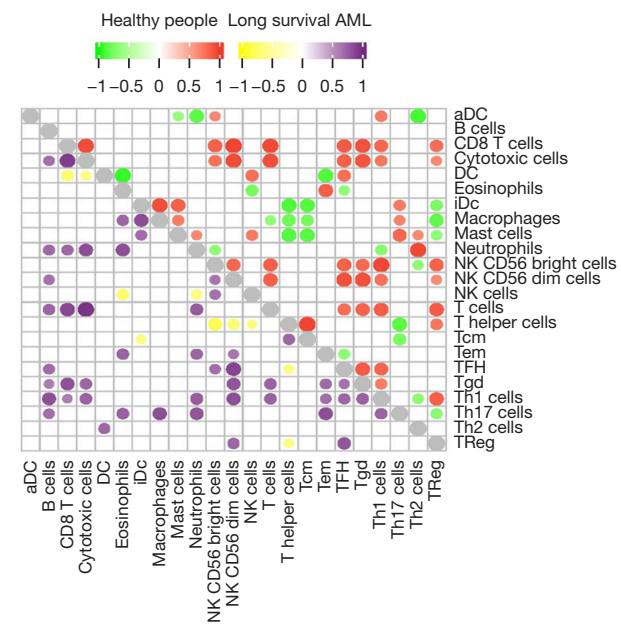

Figure 3 Immune cell analysis of two study groups. (A) Immune cell infiltration of long-term survival acute myeloid leukemia (AML) patients (discontinued chemotherapy $<3$ or $>3$ years) and healthy controls calculated by ESTIMATE, CIBERSORT, EPIC and single sample gene set enrichment analysis (ssGSEA) algorithms. (B) Immunophenotype score (IPS) of long-term survival AML patients (discontinued chemotherapy $<3$ or $>3$ years) and healthy controls. (C) Comparison of immune cell types between long-term survival AML patients (long survival AML) and healthy controls based on ssGSEA. CP, checkpoints; EC, effector cells; MHC, major histocompatibility complex; SC, suppressor cells; ssGSEA, single sample gene set enrichment analysis.

were four cluster gene expressions in AML patients with long-term survival that were highly similar to those in the healthy controls, but distinct from patients with longterm AML survival who discontinued chemotherapy after $<3$ years. Although a minority of patients with leukemia benefit from immune CP blockade therapy, a study proved that blocking the immunosuppressive microenvironment by targeting immune inhibitory receptors may hinder AML development (10). Also, a previous study suggested that the development and function of NK cells and cytotoxic T cells were inhibited in patients with AML (11). The specific mechanism remains to be elucidated.
The clinical response was found to correlate with proportions of immune cells in solid malignancies (12). AML survival was strongly associated with lymphocytes and T-lymphocyte proportion in the bone marrow (13). Reconstitution of the lymphocyte population after chemotherapy may reduce the risk of AML relapse (14).

Considering that the immune cell gene expression of patients with AML who discontinued chemotherapy after $<3$ years was different from that of the healthy controls, the antitumor cell populations in the long-term survival AML patients, such as T cells and NK cells, were assumed to have rebuilt after chemotherapy for 3 years. A further 
A

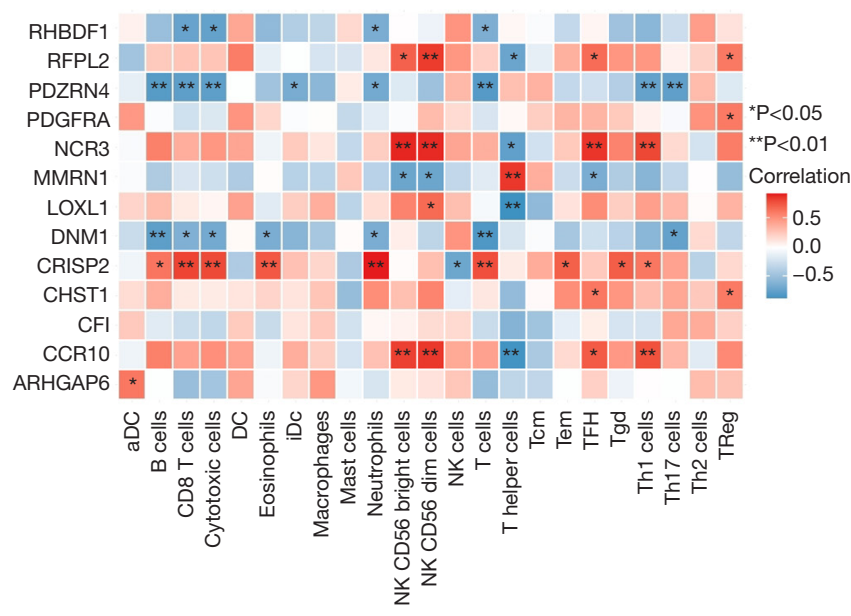

B

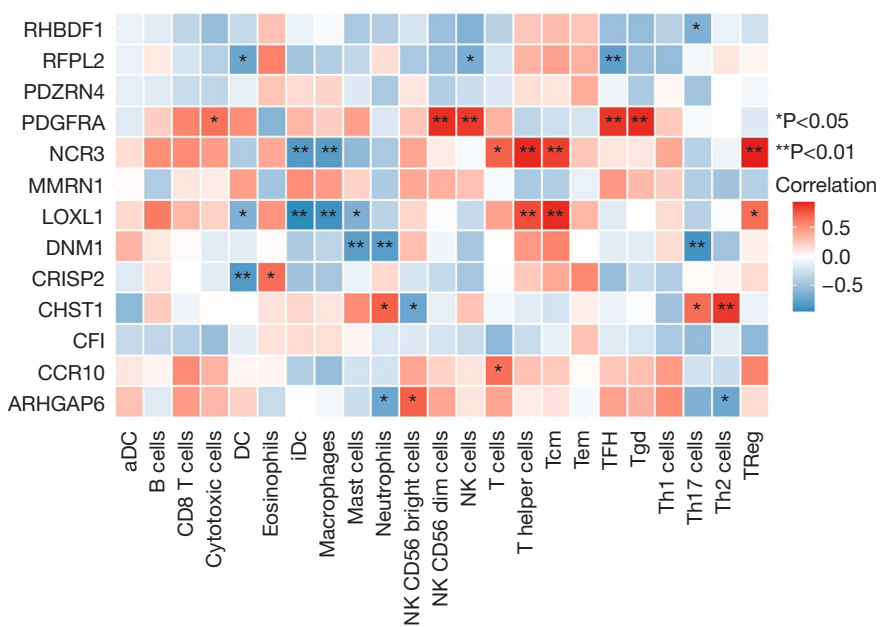

Figure 4 Relationship between immune cells and crosslinked genes of the different groups of patients. (A) Patients who discontinued chemotherapy after $<3$ years. (B) Patients who discontinued chemotherapy after $>3$ years and the healthy controls.

mechanism of immune cell reconstruction and influence on AML prognosis remains to be explored.

In summary, DNA methylation, RNA expression and TILs in patients with AML with long-term survival were slightly different than in healthy people. The profile of long-term AML survivors, especially those who discontinued chemotherapy after $<3$ years, still differed from that of healthy people. Therefore, these patients still need clinical follow-up until complete restitution of immune function is achieved.

\section{Acknowledgments}

Funding: The authors appreciate the funding of the National Key Research and Development Program of China (2019YFC0840605) and CAMS Innovation Fund for Medical Sciences (2018-I2M-AI-017).

\section{Footnote}

Reporting Checklist: The authors have completed the MDAR reporting checklist. Available at https://atm.amegroups. com/article/view/10.21037/atm-21-6681/rc

Data Sharing Statement: Available at https://atm.amegroups. com/article/view/10.21037/atm-21-6681/dss

Conflicts of Interest: All authors have completed the
ICMJE uniform disclosure form (available at https://atm. amegroups.com/article/view/10.21037/atm-21-6681/coif). The authors have no conflicts of interest to declare.

Ethical Statement: The authors are accountable for all aspects of the work in ensuring that questions related to the accuracy or integrity of any part of the work are appropriately investigated and resolved. The study was conducted in accordance with the Declaration of Helsinki (as revised in 2013). The study was approved by institutional committee of Hematology and Blood Diseases Hospital, Chinese Academy of Medical Sciences (No. KT2018091-EC-2) and written informed consent was given by all patients.

Open Access Statement: This is an Open Access article distributed in accordance with the Creative Commons Attribution-NonCommercial-NoDerivs 4.0 International License (CC BY-NC-ND 4.0), which permits the noncommercial replication and distribution of the article with the strict proviso that no changes or edits are made and the original work is properly cited (including links to both the formal publication through the relevant DOI and the license). See: https://creativecommons.org/licenses/by-nc-nd/4.0/.

\section{References}

1. Döhner H, Weisdorf DJ, Bloomfield CD. Acute Myeloid Leukemia. N Engl J Med 2015;373:1136-52. 
2. Finotello F, Trajanoski Z. Quantifying tumor-infiltrating immune cells from transcriptomics data. Cancer Immunol Immunother 2018;67:1031-40.

3. Wang Y, Cai YY, Herold T, et al. An Immune Risk Score Predicts Survival of Patients with Acute Myeloid Leukemia Receiving Chemotherapy. Clin Cancer Res. 2021;27:255-66.

4. Dong C, Zhang NJ, Zhang LJ. The Multi-Omic Prognostic Model of Oxidative Stress-Related Genes in Acute Myeloid Leukemia. Front Genet. 2021;12;722064.

5. Bindea G, Mlecnik B, Tosolini M, et al. Spatiotemporal dynamics of intratumoral immune cells reveal the immune landscape in human cancer. Immunity 2013;39:782-95.

6. Newman AM, Liu CL, Green MR, et al. Robust enumeration of cell subsets from tissue expression profiles. Nat Methods 2015;12:453-7.

7. Racle J, de Jonge K, Baumgaertner P, et al. Simultaneous enumeration of cancer and immune cell types from bulk tumor gene expression data. Elife 2017;6:26476.

8. Yoshihara K, Shahmoradgoli M, Martínez E, et al. Inferring tumour purity and stromal and immune cell admixture from expression data. Nat Commun

Cite this article as: Song Y, Hao Q, Zhang G, Fang Q, Wang Z, Li Y, Wei H, Wang Y, Jiang E, Tian Z, Jia Y, Wang M, Wang J, Mi Y. Comparison of multi-omics results between patients with acute myeloid leukemia with long-term survival and healthy controls. Ann Transl Med 2022;10(2):82. doi: 10.21037/atm-21-6681
2013;4:2612.

9. Charoentong P, Finotello F, Angelova M, et al. Pancancer Immunogenomic Analyses Reveal GenotypeImmunophenotype Relationships and Predictors of Response to Checkpoint Blockade. Cell Rep 2017;18:248-62.

10. Fridman WH, Zitvogel L, Sautès-Fridman C, et al. The immune contexture in cancer prognosis and treatment. Nat Rev Clin Oncol 2017;14:717-34.

11. Nishino M, Ramaiya NH, Hatabu H, et al. Monitoring immune-checkpoint blockade: response evaluation and biomarker development. Nat Rev Clin Oncol 2017;14:655-68.

12. Ismail MM, Abdulateef NAB. Bone marrow T-cell percentage: A novel prognostic indicator in acute myeloid leukemia. Int J Hematol 2017;105:453-64.

13. Behl D, Porrata LF, Markovic SN, et al. Absolute lymphocyte count recovery after induction chemotherapy predicts superior survival in acute myelogenous leukemia. Leukemia 2006;20:29-34.

14. Vago L, Gojo I. Immune escape and immunotherapy of acute myeloid leukemia. J Clin Invest 2020;130:1552-64. 